\title{
Two critical ratios in polymer solutions
}

\author{
T A Witten Jr† and L Schäfer $\ddagger$ \\ $\dagger$ Physics Department, University of Michigan, Ann Arbor, MI 48109, USA \\ $\ddagger$ Institut für Theoretische Physik der Universität Heidelberg, D-6900 Heidelberg 1, FRG
}

Received 19 January 1978

\begin{abstract}
The interpenetration function $\Psi$ of a dilute solution of long randomly coiling molecules may be calculated by renormalisation group methods. This quantity is of special interest since it is entirely due to excluded volume effects. Two renormalisation methods give values for the excluded volume limit of $\Psi$ which are consistent with current experimental values. This indicates that the limiting value of $\Psi$ is easier to measure than previously thought. The experimental deviations from this limit can be well represented by a simple standard corrections-to-scaling formula. We also calculate the limiting ratio of average end-to-end distance to radius of gyration of these molecules. There is a small difference from the random chain value.
\end{abstract}

\section{Introduction}

The 'excluded volume problem' concerns those aspects of the behaviour of linear random-coil molecules which are akin to a self-avoiding random walk. A few years ago de Gennes (1972) and des Cloizeaux $(1974,1975)$ discovered that a dilute solution of these molecules bears a direct relationship to a many-body system near a second-order phase transition. In particular both systems have the so called renormalisation group symmetry-their correlations transform irreducibly under a change of length scale. This 'renormalisation' approach has permitted several 'critical' properties such as the correlation length exponent $\nu$ to be calculated for the excluded volume problem.

We consider here the behaviour of the 'interpenetration function' $\Psi$ in the limit of long chains. This dimensionless parameter is essentially the second virial coefficient $A_{2}$ measured in units of the molecular weight $M$ and mean-square radius of gyration $S^{2}$ of a chaint:

$$
\Psi=\frac{1}{4 \pi^{3 / 2}} \frac{A_{2} M^{2}}{N_{\mathrm{A}} S^{3}}
$$

where $N_{\mathrm{A}}$ is Avogadro's number. This quantity deserves special attention since it is entirely due to excluded volume effects. These same excluded volume effects are responsible for the non-trivial renormalisation symmetry and thus $\Psi$ is a sensitive measure of the crucial features of the theory. The ratio $\Psi$ can be related directly to the density-density correlations of the monomer links, and hence it can be measured in a light scattering experiment. Up to the present there have been a number of non-renormalisation theories (see Yamakawa 1971, \$\$21 and 40) of $\Psi$, which differ $\dagger$ To stick to the conventional definition (see Yamakawa 1971) we have introduced a factor $\left(4 \pi^{3 / 2}\right)^{-1}$. 
qualitatively in the long-chain limit (table 1). According to the renormalisation group calculations we report below $\dagger \Psi$ should attain a value $0 \cdot 27$ within a factor of two. The uncertainty in our predictions arises from the approximation methods which assume that the dimension of space is near four. We obtain this result by two independent methods: a first-principles calculation based on our recent paper (Schäfer and Witten 1977 to be referred to as SWI), and a semi-empirical calculation based on the results of Burch and Moore (1976). These authors have calculated the dependence of the second virial coefficient $A_{2}$ and the mean square end-to-end distance $R^{2}$ on a certain interaction parameter $z$. Their work relies on an application of an approximate renormalisation group method due to Riedel and Wegner (1974). Our results differ markedly from most previous predictions, and they lie much closer to values attained thus far in experiments (see figure 1). This suggests that the asymptotic limit is readily attainable in experiments, if it has not been attained already. This would mean that a new fundamental critical ratio had been measured.

Moreover, the departures from the asymptotic value are governed by renormalisation symmetry. Indeed, the theory of corrections to scaling shows that $\Psi(M)-$ $\Psi(\infty)$ should vary for large $M$ as $M^{-\omega \nu}$, where $\omega \nu$ is calculated (Le Gillou and Zinn-Justin 1977) to be $0.465 \pm 0.01$. Available experimental data confirm this predicted $M$ dependence of $\Psi$ and improved measurements could provide much needed experimental information on the exponent $\omega$. (Current measurements of $\omega$ in phase transitions are crude (Ahlers 1977).) We stress that $\Psi(\infty)$ and $\omega$ can be directly measured using only light scattering experiments in a good solvent, without knowledge of the interaction parameter $z$.

In the process of calculating $\Psi$ we obtained a second critical ratio of interest to polymer theorists, although it is not currently measurable. This is the ratio of the mean-square end-to-end distance $R^{2}$ to the mean-square radius of gyration $S^{2}$. We obtained a value of $6 \times(1.025 \pm 0.01)$ which corroborates previous theoretical estimates (Flory 1949, Orofino and Flory 1957, Stockmayer 1960), but disagrees with numerically obtained values (Domb and Hioe 1969).

Equation (1) defines $\Psi$ for a monodisperse solution. Our theory gives directly not $\Psi$ itself, but a certain average $\langle\Psi\rangle$ measurable in polydisperse solutions (by light scattering). To obtain a prediction of $\Psi$ we thus estimate the polydispersity dependence of $\langle\Psi\rangle$ in two different ways. In one of these methods we generalise the field theory so that it is in principle able to treat monodisperse solutions directly. The other method uses a commonly employed van der Waals model.

\section{Calculation for equilibrium polydispersity}

In the standard model (Edwards 1966, Yamakawa 1971) used for excluded volume calculations a polymer consists of a set of $n$ monomer coordinates $r_{i}$, each of which is attracted to its successor $r_{i+1}$ by a harmonic potential. There is in addition a finiterange potential (the excluded volume interaction) $v\left(r_{i}-r_{j}\right)$ acting between all pairs of monomers. Following de Gennes (1972) and des Cloizeaux (1974) we assume a distribution of chain lengths $n$ governed by a monomer chemical potential $\zeta_{0}$. This amounts to assuming chemical equilibrium among chains of different lengths. The

† We reported crude calculations of this quantity in 1977 Bull. Am. Phys. Soc. 22400 and in 1978 Proc. 13th Int. Conf. on Statistical Physics, Haifa 1977 (Haifa: Israel Physical Society, Bristol: Adam Hilger). 
partition function $Z_{1}$ of the chain has the form

$$
Z_{1}=\sum_{n=1}^{\infty} \int \mathrm{d}^{d} r_{1} \ldots \mathrm{d}^{d} r_{n} \exp \left(-\mathscr{H}_{0}-\sum_{i, j} v\left(r_{i}-r_{j}\right)-\zeta_{0} n\right)
$$

where $\mathscr{H}_{0}$ contains the harmonic 'chain potentials'. Our system (see swI and des Cloizeaux 1975) consists of a grand canonical ensemble of such chains with a chain chemical potential of the form $\ln \left(h_{0}^{2}\right)$. The interaction $v$ acts between, as well as within chains.

The theory is based on a cluster expansion (see, for instance, the monograph of Yamakawa 1971) in powers of a positive interaction parameter $g_{0}$ proportional to the binary cluster integral of two monomers. This cluster expansion can be 'renormalised' to eliminate the mean distance $l$ between successive monomers. In the process of renormalisation the parameters $g_{0}$ and $\zeta_{0}$ are substituted by quantities which are derived from the values of certain correlation functions at some normalisation point. Several equivalent schemes may be found in the literature. (See, for instance, the review article of Brezin et al (1976, to be referred to as BLz).) Here we use the massless renormalisation scheme of BLZ, in which the normalisation point is chosen to be at $h_{0}=0$, average chain length $N=\infty$, and finite momentum of the correlation functions.

Invariance of the renormalised theory under change of $l$ implies scaling laws which hold for large $N$ (i.e. $N \gg 1$ ) and small average monomer density $\rho\left(\rho l^{d} \ll 1\right)$. The quantity of interest in calculating $\Psi$ is the scattering function $I_{0}(q)$, the Fourier transform of the autocorrelation of the local monomer density $\rho(x)$ :

$$
I_{0}(q) \equiv \int \mathrm{d}^{d} x \mathrm{e}^{\mathrm{i} q x}\left(\langle\rho(x) \rho(0)\rangle-\rho^{2}\right) .
$$

This function obeys the scaling law (see swI)

$$
I_{0}(q)=N_{\mathbf{R}}^{2} c_{\mathrm{p}} / j\left(Q, s_{\mathbf{R}}\right) \text {. }
$$

Here the polymer concentration $\rho / N$ is denoted by $c_{\mathrm{p}}$, the overlap $s_{\mathrm{R}}$ is defined as $l^{d} g^{*} c_{\mathrm{p}} N_{\mathrm{R}}^{\nu d}$, and the quantity $Q$ is the scaled wavenumber $q l N_{\mathrm{R}}^{\nu}$. The renormalised average chain length $N_{\mathrm{R}}$ is a fixed multiple of the physical length $N$. The 'fixed point coupling' $g^{*}$ takes the place of $g_{0}$ within the renormalised theory. This $g^{*}$ vanishes as $d \rightarrow 4$ and has the expansion (BLZ, equation (9.10); cf remark below figure 10 of BLZ)

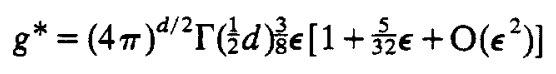

where $\epsilon=4-d$. The unrenormalised value $g_{0}$ of the binary cluster integral as well as other microscopic quantities like the length $l$ of a link influence only the scale of $N_{R}$. This scale measures the strength (for a given temperature and chemical composition) of excluded volume effects. The exponent $\nu$ occuring in these expressions can be calculated (Le Guillou and Zinn-Justin 1977) ab initio: $\nu=0.588 \pm 0.001$.

The scaling function $j\left(Q, s_{\mathrm{R}}\right)$ is calculated by renormalised perturbation theory. From our definition of $j\left(Q, s_{\mathrm{R}}\right)$ and swI, equation (40) we find

$$
j^{-1}\left(Q, s_{\mathbf{R}}\right)=\text { constant } s_{\mathbf{R}}^{-1} \mathscr{I}_{0}\left(\hat{Q}, s_{\mathrm{R}}\right)
$$

where

$$
\hat{Q}=Q\left(\frac{f(y)}{2 \partial F^{0,0}(y) / \partial y}\right)^{\nu}
$$


This function $\mathscr{I}_{0}$ can in turn be expressed by scaling functions with the help of equations (37) and (42) of SWI:

$$
\begin{gathered}
s_{\mathrm{R}}=\frac{1}{2} f(y)^{1-\nu d}\left(2 \frac{\partial F^{0,0}}{\partial y}\right)^{\nu d} \\
i^{-1}\left(Q, s_{\mathrm{R}}\right)=\text { constant } f(y)\left(\frac{\partial F^{0,0}}{\partial y}\right)^{-2}\left(\frac{\left.F^{1,1}(\hat{Q},-Q, y)\right)^{2}}{F^{0,2}(\hat{Q},-\hat{Q}, y)}-F^{2,0}(Q,-Q, y)\right) .
\end{gathered}
$$

The functions $F^{L, M}$ are scaling parts of one-line-irreducible vertex functions (see SWI, equation (28)), and $f(y)$, is related in a simple way to $F^{0,0}$ (swI, equation (36)). Expressions for $f, F^{0,0}, F^{0,2}$ can be found in the literature (see, for instance, BLZ). In the appendix we recall these results together with an outline of our calculation of $F^{1,1}$ and $F^{2,0}$. The results presented there can be combined with equations (8) and (9) to yield

$$
j\left(Q, s_{\mathrm{R}}\right)=\text { constant }\left[1+\left(1-\frac{23}{96} \epsilon\right) Q^{2}+\frac{2}{3}\left(1+\frac{9}{32} \epsilon\right) s_{\mathrm{R}}+\mathrm{O}\left(s_{\mathrm{R}}^{2}, Q^{4}, \epsilon^{2}\right)\right] .
$$

For $\epsilon=0$ equation (10) yields $j$ in the tree approximation (mean field theory), where only diagrams without closed loops are taken into account.

We now use the relation of Zimm (1948) to define the second virial coefficient $A_{2}$ and the average radius of gyration $S$ for a solution of arbitrary polydispersity:

$$
I_{0}\left(q, c_{\mathrm{p}}\right)=\text { constant } c_{\mathrm{p}} M^{2}\left(1-\frac{1}{3} q^{2} S^{2}-2 A_{2} M^{2} c_{\mathrm{p}}+\cdots\right) \text {. }
$$

We can then define the interpenetration function $\langle\Psi\rangle$ for the equilibrium ensemble. Equations (1) and (11) suggest the definition

$$
\langle\Psi\rangle \equiv \frac{N_{W}}{8(3 \pi)^{3 / 2}}\left(\frac{I_{0}}{\rho} \frac{\partial\left(\rho / I_{0}\right)}{\partial \rho}\right)\left(\frac{I_{0}}{\rho} \frac{\partial\left(\rho / I_{0}\right)}{\partial q^{2}}\right)^{-d / 2},
$$

where the weight-average chain length $N_{\mathrm{w}}$ is defined as the ratio of the second to the first moment of the chain length distribution, and the derivatives are taken with a fixed chain length distribution in the limit $q, c_{p} \rightarrow 0$. Now $\langle\Psi\rangle$ is so constructed that it can be expressed in terms of the scaling function $j\left(Q, s_{\mathrm{R}}\right)$ without any arbitrary factors. From the scaling law, equation (4), one verifies that

$$
\langle\Psi\rangle=\frac{g^{*}}{8(3 \pi)^{3 / 2}} \frac{N_{\mathrm{W}}}{N}\left(\frac{1}{j(0,0)} \frac{\partial j}{\partial S_{\mathrm{R}}}\right)\left(\frac{1}{j(0,0)} \frac{\partial j}{\partial Q^{2}}\right)^{-d / 2} .
$$

The renormalisation symmetry enters this expression in a nontrivial way (through $\boldsymbol{g}^{*}$ ) even if one uses the trivial mean field result for $j$ (equation (10)). To obtain $\langle\Psi\rangle$ to next order in $\epsilon$ we use equation (10) for $j$ and equation (5) for $g^{*}$ :

$$
8(3 \pi)^{3 / 2}(4 \pi)^{-d / 2} \Gamma^{-1}\left(\frac{1}{2} d\right)\left(N / N_{\mathrm{W}}\right)\langle\Psi\rangle=\frac{1}{4} \epsilon\left[1+\frac{11}{12} \epsilon+O\left(\epsilon^{2}\right)\right] .
$$

(Our choice of the prefactor on the left-hand side eliminates the 'kinematic' $d$ dependent prefactor in $g^{*}$ ). From the large size of the correction term we estimate a factor two uncertainty in the result.

For later use we also calculate the ratio of the radius of gyration $S$ to the end-to-end distance $R$ for an isolated chain. This ratio can be expressed in terms of $j$ 
and an analogous quantity $\left(\hat{\xi}_{\mathrm{e}}\right)^{-1}$ describing the end-point correlations (equation (A.11)):

$$
\frac{\left\langle R^{2}\right\rangle}{\left\langle S^{2}\right\rangle}=2\left(\frac{\partial}{\partial Q^{2}} \ln j(Q, 0)\right)^{-1}\left[-\frac{\partial}{\partial Q^{2}} \ln \hat{\mathscr{\xi}}_{\mathrm{e}}(Q)\right]_{Q=0}
$$

It can be calculated with the same information which allowed for the calculation of $\langle\Psi\rangle$. We find

$$
\frac{\left\langle R^{2}\right\rangle}{\left\langle S^{2}\right\rangle}=2\left[1-\frac{1}{96} \epsilon+\mathrm{O}\left(\epsilon^{2}\right)\right]
$$

\section{The influence of the chain length distribution}

Having calculated $\langle\Psi\rangle$ in the equilibrium chain length distribution we now estimate the value of $\Psi$ for a monodisperse solution. This leads us to study the dependence of $\langle\Psi\rangle$ on polydispersity. First we observe that the equilibrium length distribution has the form of a Schultz distribution whose exponent $\gamma$ is 1.1615 (Le Guillou and Zinn-Justin 1977) in three dimensions, i.e. the probability $P_{N}(n)$ that a chain has length $n$ is given by

$$
P_{\mathrm{N}}(n)=\frac{\gamma}{N \Gamma(\gamma)}\left(\gamma \frac{n}{N}\right)^{\gamma-1} \mathrm{e}^{-\gamma n / N}
$$

This may be deduced e.g. from the expression for the single chain partition function (des Cloizeaux 1974, equation (2.9)) which shows that $P_{N}(n)$ equals $z n^{\gamma-1} \exp \left[n\left(s_{c}-\right.\right.$ $s)]$. The unknown parameters $z$ and $\left(s_{c}-s\right)$ can be eliminated with the help of the relations $\Sigma_{n} P_{N}(n)=1$ and $\Sigma_{n} n P_{N}(n)=N$. The result (17) follows. For later use we note that $\gamma=1$ in four dimensions.

Equation (17) immediately yields the polydispersity dependence of $\left\langle S^{2}\right\rangle$ for a given $N$. Using the fact that $S$ for a given chain varies as its length $n$ to the power $\nu$, one obtains

$$
\left(\frac{I_{0}}{c_{\mathrm{p}}} \frac{\partial\left(c_{\mathrm{p}} / I_{0}\right)}{\partial q^{2}}\right)_{m}\left(\frac{I_{0}}{c_{\mathrm{p}}} \frac{\partial\left(c_{\mathrm{p}} / I_{0}\right)}{\partial q^{2}}\right)_{\mathrm{eq}}^{-1}=\gamma^{2 \nu} \frac{\Gamma(\gamma+2)}{\Gamma(\gamma+2+2 \nu)}
$$

where the subscripts $\mathrm{m}$ and eq stand for monodisperse and equilibrium respectively. By similar reasoning, the polydispersity ratio for the end-point distance $\left\langle R^{2}\right\rangle$ has the value

$$
\frac{\left\langle R^{2}\right\rangle_{m}}{\left\langle R^{2}\right\rangle_{e q}}=\gamma^{2 \nu} \frac{\Gamma(\gamma)}{\Gamma(\gamma+2 \nu)}
$$

The polydispersity dependence of $\left\langle A_{2}\right\rangle$ may in principle also be calculated in the renormalised theory to any given order in $\epsilon$ (Schäfer and Witten 1978). We shall content ourselves here with only the lowest order (tree) approximation, valid in the limit $\epsilon \rightarrow 0$. In that limit the scattering function has the random-phase-approximation form (Edwards 1966):

$$
I_{0}\left(q, c_{p}\right)=\frac{I^{\prime}\left(q, c_{p}\right)}{1+\bar{v} I^{\prime}\left(q, c_{p}\right)}
$$


where $\bar{v}$ is an excluded volume potential and $I^{\prime}\left(q, c_{\mathrm{p}}\right)$ is the scattering function of the solution without excluded volume effects (i.e. a superposition of random chain scattering functions (Debye 1947)):

$$
I^{\prime}\left(q, c_{\mathrm{p}}\right)=\text { constant } c_{\mathrm{p}} q^{-4} \sum_{n} P_{N}(n)\left[\mathrm{e}^{-(q l) 2 n}-1+(q l)^{2} n\right]
$$

The function $I_{0}\left(q, c_{\mathrm{p}}\right)$ should obey the scaling law, equation (4), $\dagger$ and one may thus infer that the scaling function $j$ is given by

$$
j\left(Q, s_{\mathrm{R}}\right)=\operatorname{constant}\left(\frac{2}{3} s_{\mathrm{R}}+j_{\mathrm{a}}(Q)\right)
$$

where

$$
j_{\mathrm{a}}^{-1}(Q)=Q^{-4} \sum_{n} P_{N}(n)\left[\mathrm{e}^{-Q^{2 n / N}}-1+Q^{2}(n / N)\right] .
$$

Note that $Q^{2}=\left(q I N_{R}^{\nu}\right)^{2}=q^{2} l^{2} N$ in four dimensions. In deriving equation (21) we have eliminated the parameter $\bar{v}$ by comparing with our previous expression for $j$ (equation (10)) taking $\epsilon \rightarrow 0$ and $P_{N}(n)=(1 / N) \exp (-n / N)$ which is the form of the equilibrium chain length distribution in four dimensions. Now using equation (17) for $P_{N}$ we find the influence of polydispersity on the concentration derivative of $I_{0}$ :

$$
\left(\frac{I_{0}}{\rho} \frac{\partial\left(\rho / I_{0}\right)}{\partial \rho}\right)_{\mathrm{m}}\left(\frac{I_{0}}{\rho} \frac{\partial\left(\rho / I_{0}\right)}{\partial \rho}\right)_{\mathrm{eq}}^{-1}=\frac{\gamma}{\gamma+1} \equiv\left(\frac{N}{N_{\mathrm{w}}}\right)_{\mathrm{eq}} .
$$

Again, this result is exact in the limit $d \rightarrow 4$, where $\gamma \rightarrow 1$.

The effect of polydispersity on $\left\langle A_{2}\right\rangle$ can be estimated independently with a simple van der Waals model suggested by the form of the scaling law (equation (4)). One assumes that each molecule behaves like a hard sphere whose radius varies as $n^{\nu}$. We have evaluated the $\left\langle A_{2}\right\rangle$ ratio of equation (23) in this model (see Yamakawa 1971 p 220, or Casassa 1962). In three dimensions, the $\left\langle A_{2}\right\rangle$ ratio is 0.524 in the hard sphere model, as compared to 0.537 from equation (23). (In four dimensions $\left(\gamma=1, \nu=\frac{1}{2}\right)$ the hard-sphere model gives a ratio $10 \%$ less than the exact result of $0 \cdot 5$.

Now we may use our estimates equations (18) and (23) to evaluate $\Psi$ for a monodisperse solution:

$$
\Psi=\frac{N}{N_{\mathrm{W}}}\langle\Psi\rangle_{\epsilon=1} \times(0 \cdot 299)^{-3 / 2} \times 0.537 .
$$

Taking $\left(N / N_{\mathrm{W}}\right)\langle\Psi\rangle$ from equation (14) we find

$$
\Psi=0 \cdot 268 \pm 100 \% \text {. }
$$

The ratio $R^{2} / S^{2}$ may be found using equation (16) for $\left\langle R^{2}\right\rangle /\left\langle S^{2}\right\rangle$ and the exact correction factors equations (18) and (19). The result, correct to order $\epsilon$, is

$$
\frac{R^{2}}{S^{2}}=2 \frac{(\gamma+2 \nu)(\gamma+2 \nu+1)}{\gamma(\gamma+1)}\left(1-\frac{\epsilon}{96}+\mathrm{O}\left(\epsilon^{2}\right)\right)=6 \times 1.025 \pm 1 \% .
$$

The factor 6 in this result is just the ratio of $R^{2}$ to $S^{2}$ for non-interacting random

$\dagger$ That the scaling law holds in the same form for general polydispersity is a new result (Schäfer and Witten 1978).

$\ddagger$ Note that Yamakawa's $\nu$ is our $2-\nu d=0.236$ and that his $h$ is our $\gamma=1.1615$. 
chains and is independent of the dimension $d$ of space (Yamakawa 1971, equation (7.17)). For self-avoiding chains the ratio is slightly but significantly altered. Our result for $R^{2} / S^{2}$ disagrees with previous values obtained numerically (Domb and Hioe $1969)$; the numerical values lie much further from the non-interacting chain value.

\section{Alternative calculation of $\Psi$}

In their recent paper Burch and Moore (1976) calculated some quantities closely related to $\Psi$ using the semi-phenomenological approach of Riedel and Wegner (1974). Their work may be extended to obtain $\Psi$ and the value thus obtained agrees with our result (equation (25)). Specifically, Burch and Moore calculated in a monodisperse ensemble the dimensionless second virial coefficient $X(z)$ where $z$ is a widely used interaction parameter proportional to our $g_{0}$ and to $N^{1 / 2}$ :

$$
X(z)=\frac{A_{2}(N, z)}{\left(\partial A_{2}(N, z) / \partial z\right)_{z=0}} .
$$

They also calculated the end-to-end expansion factor $\alpha_{R}$ (their $\alpha$ ) defined by

$$
\alpha_{R}^{2}(z)=R^{2}(N, z) / R^{2}(N, 0)
$$

From these, in the large- $z$ (long-chain) limit one may obtain a quantity closely related to $\Psi$. Indeed, $\Psi$ and the interaction $z$ are defined so that

$$
\Psi=\lim _{z \rightarrow \infty} \alpha_{s}^{-3}(z) X(z)
$$

where $\alpha_{S}$ is the expansion factor for the radius of gyration $S$ (Yamakawa 1971, equations (21.17), (21.5), (13.32)). By combining the Burch and Moore expressions for $X(z)$, equation (4.25) and equation (5.7), we may obtain the analogous quantity

$$
\Psi_{\mathrm{e}}=\lim _{z \rightarrow \infty} \alpha_{R}^{-3}(z) X(z)=0 \cdot 208
$$

In their calculation Burch and Moore have adjusted an (arbitrary) scale factor for $X(z)$ to assure that $X(z)=z$ for small $z$, and a scale factor for $z$ has been set so that $d^{2} X / d z^{2}$ has the correct value (Yamakawa 1971, equation (21.7)) at $z=0$. Now these scale factors may be chosen in alternative ways, and a range of $\Psi_{e}$ values results. Thus, if the scale of $z$ is adjusted to match $\alpha_{R}$ rather than $d^{2} X / d z^{2}$ at $z \rightarrow 0$ then $\Psi_{\mathrm{e}}=0 \cdot 114$; if $z$ is adjusted to match $\Psi_{\mathrm{e}}(z)$ itself for small $z$, then $\Psi_{\mathrm{e}}=0 \cdot 169$.

These three $\Psi_{\mathrm{e}}$ values are consistent with each other within a factor of two. They are also consistent with our value of $\Psi$. In fact from the definitions of $\Psi$ and $\Psi_{\mathrm{e}}$, we expect

$$
\frac{\Psi}{\Psi_{\mathrm{e}}}=\left(\frac{\alpha_{R}}{\alpha_{S}}\right)^{3}=\left(\frac{1}{6} \frac{R^{2}}{S^{2}}\right)^{3 / 2} .
$$

From equation (26) this ratio is about $1 \cdot 04$, so that $\Psi$ should be roughly equal to $\Psi_{\mathrm{e}}$. Now the $\Psi_{\mathrm{e}}$ values based on the paper of Burch and Moore do lie in the same range as our value from equation (25). The two independent calculations thus corroborate each other. 


\section{Comparison with experiment}

We compare our results with the light scattering data of Berry (1966) and Norisuye et al (1968). Their results have been analysed to give the interpenetration function as a function of the cube of the swelling factor, $\alpha_{s}^{3}$ (see Norisuye et al 1968, Yamakawa 1971). Our calculation has been concerned only with the excluded volume limit of the function $\Psi\left(\alpha_{s}^{3}\right)$. The theory of corrections to scaling (Wegner 1972a, b, BLz, equation (8.6)) can be used to determine the form of the leading correction near $\alpha_{S}=\infty$. According to this theory for large $N$ we expect

$$
\Psi(N)-\Psi(\infty) \sim N^{-\omega \omega} \text {. }
$$

On the other hand, the expansion factor $\alpha_{S}$ varies as $N^{\nu-1}$ in the excluded volume limit (swI, equation (50)). Combining these relations and assuming that $\Psi$ is a universal function of $\alpha_{S}$ (Norisuye et al 1968, Burch and Moore 1976) we find for $\alpha_{S} \gg 1$,

$$
\Psi\left(\alpha_{S}^{3}\right)-\Psi(\infty) \approx-\Psi_{1} \alpha_{S}^{-\omega \nu /\left(\nu-\frac{1}{2}\right)}
$$

where $\Psi_{1}$ is a universal constant. Using the results for $\omega \nu$ and $\nu$ quoted above, the power of $\alpha_{S}^{3}$ is found to be $-1 \cdot 67$. Figure 1 shows a crude fit of Berry's data on $\Psi\left(\alpha_{S}^{3}\right)$ using equation (33) with $\dagger(\infty)=\Psi_{1}=0.315$. We note that the data can be fitted surprisingly well by this simple form. Fitting the Norisuye et al data in the same way, we obtain an equally good fit, using $\Psi(\infty)=\Psi_{1}=0 \cdot 28$. It is clear that the data are completely consistent with our estimates for $\Psi(\infty)$, i.e. $0 \cdot 27 \pm 100 \%$.

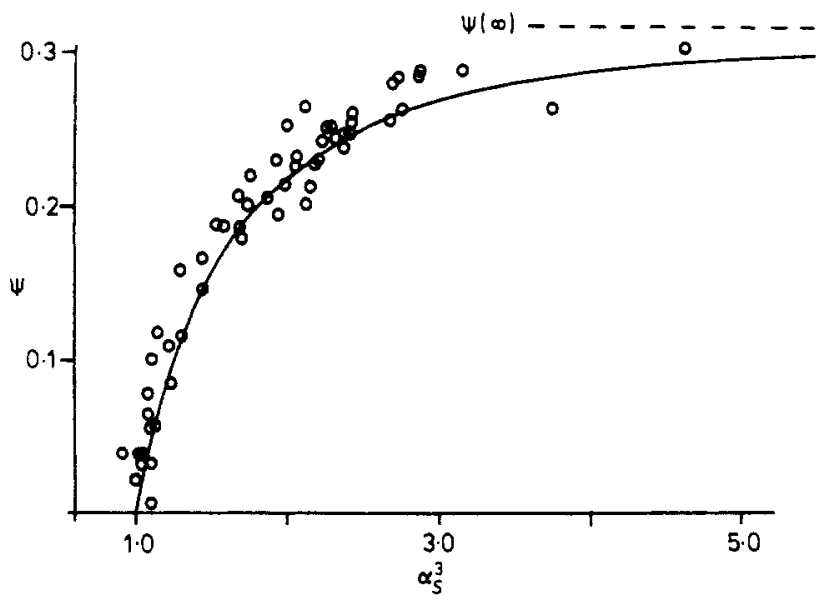

Figure 1. Interpenetration function $\Psi$ against $\alpha_{s}^{3}$, the cube of the expansion factor. Open circles are data on polystyrene in various solvents (Berry 1966), as given by Yamakawa (1971). The full curve is given by equation (33) with $\Psi(\infty)=\Psi_{1}=0.315$.

We have not tried to extract an experimental value for $\omega$ from the data: the experimental uncertainties are too large. A part of these uncertainties is due to polydispersity effects. The ratio of the weight average $N_{\mathrm{w}}$ to the number average $N$ of

† The data are not accurate enough to allow for an evaluation of both parameters $\Psi(\infty)$ and $\Psi_{1}$ independently. The constraint $\Psi(\infty)=\Psi_{1}$ yields an overall fit, which is quite good even outside the asymptotic region. 
the chain length varies from 1.01 to 1.07 in Berry's samples and from 1.11 to 1.21 in those of Norisuye et al. Assuming a Schultz distribution and using mean field theory (equations (18) and (23)) we can estimate the influence on $\langle\Psi(\infty)\rangle$ of this change in polydispersity. We find that the dependence of $(\Psi(\infty)\rangle$ on $N_{\mathrm{W}} / N$ is fairly strong:

$$
\left.\frac{\partial\langle\Psi(\infty)\rangle}{\partial N_{\mathrm{W}} / N}\right|_{N_{\mathrm{W}} / N=1}=2-\frac{3}{2} \nu(3+2 \nu) \sim-1.7 \text {. }
$$

This suggests that polydispersity effects can account for about $10 \%$ to $15 \%$ spread in the $\Psi$-values of figure 1 .

Most previous theoretical interpretations of these data give a qualitatively different picture from the above. In table I we list the predictions of the three self-consistent theories treated in Yamakawa's book (\$40). The three give widely different behaviour, and all suggest that the currently measured $\Psi$ values are far below any asymptotic limit. All three are more or less consistent with the experimental data (see Yamakawa 1971, figures VIII.11 and VIII.12). We have also listed two other theoretical values of $\Psi(\infty)$, which are close to the measured values. Finally, we have summarised the results of the present work obtained by using the renormalisation group (RG) symmetry of the system. The two RG calculations are consistent with each other and with the data. They are inconsistent with the three self-consistent theories and instead indicate strongly that the current data have attained the asymptotic $\Psi$-value within a few per cent. The RG calculations also suggest that data on $\Psi\left(\alpha_{s}^{3}\right)$ or $\Psi(N)$ with some refinement could yield improved measurements of the critical exponent $\omega$. The question to be settled now is whether the data have attained the asymptotic value or not.

Table 1. Asymptotic values of $\Psi\left(\alpha^{3}\right)$ for several theories.

\begin{tabular}{ll}
\hline Method & $\Psi\left(\alpha^{3}\right)$ \\
\hline FO & $0.29 \ln \alpha^{3}$ \\
FM & $0.12 \ln \alpha^{3}$ \\
KYT & $0.784+O\left(\left(\alpha^{3}\right)^{-0.21}\right)$ \\
\hline K & 0.20 \\
FCM & 0.18 \\
\hline equation $(25)$ & $0.27 \pm 100 \%+O\left(\left(\alpha^{3}\right)^{-1.67}\right)$ \\
$\quad\left(A_{2}\right)$ & 0.208 \\
BM $(\Psi)$ & 0.169 \\
$\left(\alpha_{R}\right)$ & 0.114 \\
\hline
\end{tabular}

FO; Orofino and Flory (1957); FM; Stockmayer (1960); KYT, Yamakawa and Tanaka (1967); K, Kurata; FCM, Fixmann, Casassa, Markovitz. The values 'K' and 'FCM' are taken from figure IV.5 of Yamakawa's monograph. Equation (25) and BM refer to this paper. BM: based on the Burch and Moore method adjusted at small $z$ to match the quantity given in parentheses.

\section{Acknowledgment}

The authors wish to thank Professor M A Moore for helpful correspondence. 


\section{Appendix. Expressions for the scaling functions}

The scaling functions are to be calculated by perturbation theory based on a renormalised Lagrangian density. We follow closely BLZ and we use the massless renormalisation scheme explained there. The scaling functions emerge naturally in terms of the 'magnetic' scaling variables (see swi, equations $\left(28^{\prime}\right),\left(28^{\prime \prime}\right)$ )

$$
\begin{aligned}
& \hat{Q}=\left(g^{*} M^{2}\right)^{-\nu / 2 \beta} q \\
& y=t\left(g^{*} M^{2}\right)^{-1 / 2 \beta}
\end{aligned}
$$

where the last quantity is called $x$ in BLz (above equation (9.25)). The quantities $t$ and $M$ are the renormalised temperature and magnetisation, respectively.

The scaling function $f(y)$ can be taken directly from BLz, equation (9.25):

$f(y)=y+\frac{1}{6}+\frac{3}{8} \epsilon\left\{\frac{1}{2}\left(y+\frac{1}{2}\right)\left[\ln \left(y+\frac{1}{2}\right)+1\right]-\frac{1}{6}\left(y+\frac{1}{6}\right)\left[\ln \left(y+\frac{1}{6}\right)+1\right]\right\}+O\left(\epsilon^{2}\right)$.

Note that the polymer system corresponds to a zero-component field theory (i.e. $n=0$ in BLz). The expressions for $F^{0.0}$ and $F^{0.2}$ follow easily from BLZ, equations (9.24) and (9.46), setting $g=g^{*}, g^{*} M^{2}=1, t=$ our $y, p^{2}=\hat{Q}^{2}$, and $i=j=1$ :

$$
\begin{aligned}
2 F^{0,0}(y)=y+ & \frac{1}{12}+\frac{3}{32} \epsilon\left[\left(y+\frac{1}{2}\right)^{2}-\left(y+\frac{1}{6}\right)^{2}+2\left(y+\frac{1}{2}\right)^{2} \ln \left(y+\frac{1}{2}\right)\right. \\
& \left.-2\left(y+\frac{1}{6}\right)^{2} \ln \left(y+\frac{1}{6}\right)\right]+O\left(\epsilon^{2}\right) \\
F^{0,2}(\hat{Q},-\hat{Q}, y) & \\
= & y+\frac{1}{2}+\frac{1}{16} \epsilon\left[2 y+\frac{20}{3}-\left(y+\frac{1}{2}\right) \ln \left(y+\frac{1}{6}\right)+3\left(y+\frac{3}{2}\right) \ln \left(y+\frac{1}{2}\right)\right] \\
& +\hat{Q}^{2}\left\{1+\frac{1}{32} \epsilon\left[\left(y+\frac{1}{2}\right)^{-1}-\frac{1}{9}\left(y+\frac{1}{6}\right)^{-1}\right]\right\}+O\left(\hat{Q}^{4}, \epsilon^{2}\right) .
\end{aligned}
$$
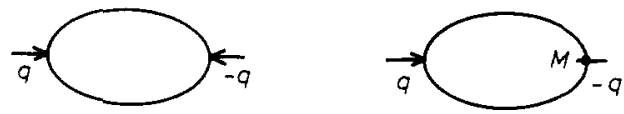

Figure 2. One-loop contributions to: (a) $\Gamma^{2.0}$; and (b) $\Gamma^{1,1}$. The arrows indicate $S^{2}$ insertions. The propagators contain both longitudinal and transverse parts.

The diagrams contributing to $F^{2,0}$ and $F^{1,1}$ are given in figure 2. They yield

$$
\begin{aligned}
& D_{(a)}=-\frac{1}{2} S\left(\mathscr{I}\left(a_{1}\right)-\mathscr{I}\left(a_{2}\right)\right) \\
& D_{(b)}=\frac{1}{6} M g^{*} S\left(2+\ln q^{2}+3 \mathscr{I}\left(a_{1}\right)-\mathscr{\Phi}\left(a_{2}\right)\right)
\end{aligned}
$$

where

$$
\begin{aligned}
& \mathscr{F}(a)=a \ln a-(a-1) \ln (a-1)-1 \\
& S=2(4 \pi)^{-d / 2}\left(\Gamma\left(\frac{1}{2} d\right)\right)^{-1}
\end{aligned}
$$

and

$$
a_{i}=\frac{1}{2}+\frac{1}{2}\left\{1+4 q^{-2}\left[Z+g^{*} \frac{1}{6} M^{2}\left(1+2 \delta_{i 1}\right)\right]\right\}^{1 / 2}
$$


Since to one-loop order $\Gamma^{1,1}=M+D_{(b)}, \Gamma^{2,0}=D_{(a)}$ we find the following results for the scaling functions (see swI, equation (28)):

$$
\begin{aligned}
& F^{1,1}(\hat{Q},-\hat{Q}, y) \\
& =1+\frac{1}{16} \epsilon\left[4+3 \ln \left(y+\frac{1}{2}\right)-\ln \left(y+\frac{1}{6}\right)\right] \\
& +\frac{1}{32} \epsilon \hat{Q}^{2}\left[\left(y+\frac{1}{2}\right)^{-1}-\frac{1}{3}\left(y+\frac{1}{6}\right)^{-1}\right]+O\left(\hat{Q}^{4}, \epsilon^{2}\right) \\
& F^{2,0}(\hat{Q},-\hat{Q}, y) \\
& =-\frac{3}{16} \epsilon\left[\ln \left(y+\frac{1}{2}\right)-\ln \left(y+\frac{1}{6}\right)\right] \\
& -\frac{1}{32} \epsilon \hat{Q}^{2}\left[\left(y+\frac{1}{2}\right)^{-1}-\left(y+\frac{1}{6}\right)^{-1}\right]+\mathrm{O}\left(\hat{Q}^{4}, \epsilon^{2}\right) \text {. }
\end{aligned}
$$

These results allow for the calculation of $j\left(Q, s_{R}\right)$ to the order considered here $t$.

To define the end-to-end distance $R$ in the single chain limit we can consider the correlations among the ends of arbitrary chains. This correlation function is given by (see SWI, equations (43) and (45))

$$
I_{\mathrm{e}}\left(q, c_{\mathrm{p}}, N\right)=\mathrm{constant} c_{\mathrm{p}}^{2} N_{\mathrm{R}}^{\nu d}\left(\frac{f(y)}{2 \partial F^{0,0} / \partial y}\right)^{\nu d}\left(F^{0,2}(\hat{Q},-\hat{Q}, y)\right)^{-1}
$$

The function $\hat{\mathscr{F}}_{\mathrm{e}}(Q)$ occurring in equation (15) is defined as the single-chain limit of $I_{\mathrm{e}}$ :

$$
\hat{\mathscr{\rho}}_{\mathrm{e}}(Q)=\text { constant } \lim _{c_{\mathrm{P}} \rightarrow 0} \frac{1}{c_{\mathrm{p}}} I_{e}\left(q, c_{\mathrm{p}}, N\right)
$$

(compare swI, equation (47)).

\section{References}

Ahlers G 1977 Quantum Liquids eds J Ruvolds and T Regge (Amsterdam: North-Holland) to be published Berry G C 1966 J. Chem. Phys. 494550

Brézin E, Le Guillou J C and Zinn-Justin J 1973 Phys. Rev. D 82418

- 1976, Phase Transitions and Critical Phenomena, vol. 6 p 125 eds C Domb and M S Green (New York: Academic)

Burch D J and Moore M A 1976 J. Phys. A: Math. Gen. 9435

Casassa E F 1962 Polymer 3625

des Cloizeaux J 1974 Phys. Rev. A 101665

1975 J. Physique 36281

Debye P 1947 J. Phys. Colloid Chem. 5118

Domb C and Hioe F T 1969 J. Chem. Phys. 511915

Edwards S F 1966 Proc. Phys. Soc. 88265

Flory P J 1949 J. Chem. Phys. 17303

de Gennes P G 1972 Phys. Lett 38A 339

Le Guillou J C and Zinn-Justin J 1977 Phys. Rev. Lett. 3995

Norisuye T, Kawahara K, Teramoto A and Fujita H 1968 J. Chem. Phys. 494330

Orofino T A and Flory P J 1957 J. Chem. Phys. 261067

$\dagger$ In deriving $j$ for small $s_{\mathbf{R}}$ (large $y$ ), one finds divergent behaviour in $y$ in the intermediate stages; the formulae in $y$ are there to be interpreted in the limit $\epsilon \rightarrow 0$, with $y$ large but fixed. The divergences vanish once the $y$ is eliminated in favour of $s_{R}$. They are an artifact of the form of the scaling laws given in BLZ, des Cloizeaux (1975) and SWI. Here we have used the laws in this form purely to give a compact presentation. The function $i$ may also be expressed in terms of other scaling functions, from which the leading power in $t$ rather than $M$ has been extracted (as in BLz, equation (6.55)). Then each order in $\epsilon$ is manifestly analytic in the limit of interest. 
Riedel E K and Wegner F J 1974 Phys. Rev. B 9294

Schäfer L and Witten T A $1977 \mathrm{~J}$. Chem. Phys. 662121

1978 in preparation

Stockmayer W H 1960 Makromolek. Chem. 3554

Wegner F J 1972a Phys. Rev. B 54528 1972b Phys. Rev. B 61891

Yamakawa H 1971 Modem Theory of Polymer Solutions (New York: Harper and Row)

Yamakawa H and Kurata M 1957 J. Chem. Phys. 321852

Yamakawa H and Tanaka G 1967 J. Chem. Phys. 473991

Zimm B H 1948 J. Chem. Phys. 161093 